\title{
Mechanical thrombectomy in acute ischemic stroke with tandem occlusions: impact of extracranial carotid lesion etiology on endovascular management and outcome
}

\author{
Valerio Da Ros, PhD, ${ }^{1}$ Jacopo Scaggiante, MD, ${ }^{1,13}$ Francesca Pitocchi, MD, ${ }^{1}$ \\ Fabrizio Sallustio, MD, ${ }^{2}$ Simona Lattanzi, MD, ${ }^{3}$ Giuseppe Emmanuele Umana, MD, ${ }^{4}$ \\ Bipin Chaurasia, MD, ${ }^{5}$ Monica Bandettini di Poggio, MD, ${ }^{6}$ Gianpaolo Toscano, MD, ${ }^{7}$ \\ Claudia Rolla Bigliani, MD, ${ }^{8}$ Maria Ruggiero, MD, ${ }^{9}$ Nicolò Haznedari, MD, ${ }^{9}$ \\ Alessandro Sgreccia, MD, ${ }^{10}$ Giuseppina Sanfilippo, MD, ${ }^{11}$ Marina Diomedi, MD, ${ }^{12}$ \\ Cinzia Finocchi, MD, ${ }^{6}$ and Roberto Floris, $\mathrm{PhD}^{1}$
}

1Department of Biomedicine, Interventional Neuroradiology Unit, University of Rome Tor Vergata, Rome; ${ }^{2}$ Comprehensive Stroke Center, Tor Vergata University Hospital, Rome; ${ }^{3}$ Neurological Clinic, Department of Experimental and Clinical Medicine, Marche Polytechnic University, Ancona; ${ }^{4}$ Department of Neurosurgery, Trauma Center, Gamma Knife Center, Cannizzaro Hospital, Catania, Italy; ${ }^{5}$ Department of Neurosurgery, Bangabandhu Sheikh Mujib Medical University, Dhaka, Bangladesh; ${ }^{6}$ Ospedale Policlinico San Martino, Department of Neurosciences, Rehabilitation, Ophthalmology, Genetics, Maternal and Child Health (DINOGMI), University of Genova; ${ }^{7}$ Stroke Unit Policlinico San Matteo, IRCCS Mondino Fundation, Pavia; ${ }^{8}$ Department of Diagnostic and Interventional Neuroradiology, Policlinico Universitario San Martino, Genova; ${ }^{2}$ Neuroradiology Unit, AUSL Romagna, Cesena; ${ }^{10}$ Department of Clinical Neuroradiology and Interventional Neuroradiology, AOU Ospedali Riuniti, Ancona; ${ }^{11}$ Department of Diagnostic and Interventional Radiology and Neuroradiology, IRCCS Policlinico San Matteo, Pavia; ${ }^{12}$ Department of Systems Medicine, University of Rome Tor Vergata, Rome, Italy; and ${ }^{13}$ Medical University of South Carolina, Division of Neuroradiology, Charleston, South Carolina

OBJECTIVE Different etiologies of extracranial internal carotid artery steno-occlusive lesions (ECLs) in patients with acute ischemic stroke (AIS) and tandem occlusion (TO) have been pooled together in randomized trials. However, carotid atherosclerosis (CA) and carotid dissection (CD), the two most common ECL etiologies, are distinct nosological entities. The authors aimed to determine if ECL etiology has impacts on the endovascular management and outcome of patients with $\mathrm{TO}$.

METHODS A multicenter, retrospective study of prospectively collected data was conducted. AIS patients were included who had TO due to internal CA or CD and ipsilateral $\mathrm{M}_{1}$ middle cerebral artery occlusion and underwent endovascular treatment (EVT). Comparative analyses including demographic data, safety, successful recanalization rates, and clinical outcome were performed according to EVT and ECL etiology.

RESULTS In total, 214 AIS patients with TOs were included (77.6\% CA related, $22.4 \%$ CD related). Patients treated with a retrograde approach were more often functionally independent at 3 months than patients treated with an antegrade approach (OR 0.6, 95\% Cl 0.4-0.9). Patients with CD-related TOs achieved 90-day clinical independence more often than patients with CA-related TOs (OR 1.4, 95\% Cl 1.1-2.0). Emergency stenting use was associated with good 3-month clinical outcome only in patients with CA-related TOs (OR 1.4, 95\% Cl 1.1-2.1). Symptomatic intracranial hemorrhage (sICH) occurred in $10.7 \%$ of patients, without differences associated with ECL etiology.

ABBREVIATIONS AIS = acute ischemic stroke; ASPECTS = Alberta Stroke Program Early Computed Tomography Score; $C A=$ carotid atherosclerosis; $C D=$ carotid dissection; EASI = Endovascular Acute Stroke Intervention; ECL = extracranial internal carotid artery steno-occlusive lesion; EVT = endovascular treatment; ICH = intracranial hemorrhage; IVT = intravenous thrombolysis; MR CLEAN = Multicenter Randomized Clinical Trial of Endovascular Treatment for Acute Ischemic Stroke in the Netherlands; mRS = modified Rankin Scale; $\mathrm{MT}=$ mechanical thrombectomy; $\mathrm{mTICI}=$ modified Thrombolysis in Cerebrovascular Infarction; NASCET = North American Symptomatic Carotid Endarterectomy Trial; NIHSS = National Institutes of Health Stroke Scale; $\mathrm{SICH}=$ symptomatic ICH; TITAN = Thrombectomy in Tandem Lesions; TO = tandem occlusion.

SUBMITTED February 24, 2021. ACCEPTED April 7, 2021.

INCLUDE WHEN CITING DOI: 10.3171/2021.4.FOCUS21111. 
CONCLUSIONS ECL etiology impacts both EVT approach and clinical outcome in patients with TOs. Patients with CD-related TO achieved higher 3-month functional independence rates than patients with CA-related TOs. A retrograde approach can be desirable for both CA- and CD-related TOs, and emergency stenting is likely better justified in CArelated TOs.

https://thejns.org/doi/abs/10.3171/2021.4.FOCUS21111

KEYWORDS tandem occlusions; acute ischemic stroke; thrombolysis; carotid atherosclerosis; carotid dissection; thrombectomy; endovascular acute stroke intervention

$\mathrm{T}$ ANDEM occlusions (TOs) are challenging lesions that occur in up to $16 \%-20 \%$ of patients presenting with acute ischemic stroke (AIS) of the anterior circulation. Poor antegrade blood flow and large thrombus burden contribute to the modest efficacy of intravenous thrombolysis (IVT), with clinical improvement obtained in only $25 \%$ of cases. ${ }^{1}$ Endovascular treatment (EVT) has shown good outcomes in half of treated patients. ${ }^{2}$ The two most frequent causes of extracranial internal carotid artery steno-occlusive lesions (ECLs) underlying TOs are internal carotid atherosclerosis (CA) and carotid dissection (CD). CD comprises $20 \%-25 \%$ of stroke in young patients, ${ }^{3}$ but it is rarely responsible for hemodynamic stroke and stroke recurrence. ${ }^{4}$ Conversely, CA-related TOs generally affect older patients with chronic and segmental carotid lesions. The composition of clots that originate from CA lesions and migrate into intracranial arteries is variable, ${ }^{5,6}$ and the stroke recurrence rate from these clots is high. ${ }^{7}$ Although $\mathrm{CA}$ and CD are distinct entities with specific pathophysiological characteristics, evaluation of these conditions individually has been limited. To our knowledge, comparison between CA- and CD-related TOs has been previously reported in only two observational studies, from the Thrombectomy in Tandem Lesions (TITAN) investigators and the Multicenter Randomized Clinical Trial of Endovascular Treatment for Acute Ischemic Stroke in the Netherlands (MR CLEAN) registry. ${ }^{8,9}$ Moreover, despite similar rates of successful reperfusion obtained in $\mathrm{CA}$ and $\mathrm{CD},{ }^{10}$ an increased understanding of the distinction between these clinical entities might influence clinical strategies and lead to new technical EVT considerations. ${ }^{5}$ The Endovascular Acute Stroke Intervention (EASI) trial demonstrated that randomization of patients with TOs is possible,,$^{11}$ but to date robust data and recommendations about optimal ECL management are lacking. This limited amount of information is reflected in the most recent American ${ }^{12}$ and Europe$\mathrm{an}^{13}$ guidelines, with data from randomized trials on TOs like TITAN (NCT03978988) ${ }^{14}$ and the EASI-Tandem Occlusion (EASI-TOC) study, which are still ongoing. ${ }^{15}$ The aim of this multicenter study was to explore the impact of the nature of cervical carotid artery lesions, namely CD versus $\mathrm{CA}$, on endovascular management and outcome in patients with AIS and TOs.

\section{Methods \\ Study Design}

Between January 2018 and January 2020, we retrospectively identified consecutive patients with AIS who were admitted at 5 Italian neuro-interventional tertiary stroke centers and underwent EVT. Patients with anterior circulation TO due to internal CA or $\mathrm{CD}$ and ipsilateral intracranial $\mathrm{M}_{1}$ middle cerebral artery occlusion, CT Al- berta Stroke Program Early Computed Tomography Score (ASPECTS) $>5$, and premorbid modified Rankin Scale (mRS) score $<2$ were included in this analysis. ECL was defined as complete occlusion or $\geq 90 \%$ stenosis according to the North American Symptomatic Carotid Endarterectomy Trial (NASCET) ${ }^{16}$ criteria confirmed at initial cerebral angiography. The ECL cause was defined according to its morphology on angiograms. Patients with unclear or other causes of carotid disease were excluded. No ethics committee approval was required for this retrospective observational study.

\section{Outcome Measures}

We collected data for the following variables: demographic characteristics (i.e., age, sex), cardiovascular risk factors (i.e., atrial fibrillation, arterial hypertension, diabetes mellitus, hypercholesterolemia, smoking, previous cardio-cerebrovascular accidents), clinical and radiological assessments (i.e., mRS score, National Institutes of Health Stroke Scale [NIHSS] score, imaging characteristics [i.e., ASPECTS]), stroke side, completeness of the circle of Willis, hemorrhagic transformation, antegrade or retrograde recanalization approach, procedural time, (i.e., onset-to-groin time, onset-to-recanalization time), number of mechanical thrombectomy (MT) attempts, and ECL treatment. ECL treatments included carotid angioplasty only, thrombo-aspiration via a large-bore catheter to pass the lesion only, or emergency carotid stenting. Preprocedural, procedural, 24- to 48-hour postprocedural, discharge, and 3-month clinical data were prospectively collected.

The primary outcome measure was 90 -day functional independence defined as an $\mathrm{mRS}$ score $\leq 2$; favorable clinical outcome was also considered and defined as 90-day $\mathrm{mRS}$ score $\leq 3$. Secondary outcomes included successful reperfusion (modified Thrombolysis in Cerebrovascular Infarction [mTICI] scores of $2 b-3$ ), complete reperfusion (mTICI score of 3), all-cause 90-day mortality, any procedural-related complications, including symptomatic intracranial hemorrhage (sICH), and thromboembolism.

\section{Imaging Evaluation}

All CT scans were obtained with 64 slices using helicoid acquisition (GE LightSpeed VCT 64; GE Healthcare). At each institution, two neuro-interventional radiologists independently analyzed preprocedural CT scans. Any disagreement was resolved by consensus with a third neuroradiologist (i.e., the head of the department). CT angiography was used to assess completeness of the circle of Willis, defined as the presence of both the anterior and posterior communicating arteries and carotid patency, including the presence of significant stenosis of the contralateral carotid according to the NASCET criteria. ${ }^{16}$ 
$\mathrm{CD}$ was recognized on angiograms by the presence of a tapered or flame-shaped stenosis or occlusion occurring $>2 \mathrm{~cm}$ from the internal carotid origin, extending to or not near the skull base.${ }^{17} \mathrm{CA}$ was identified as a stenosis or occlusion at or near the internal carotid origin associated or not with adjacent calcified plaque.

Follow-up control CT or MRI was performed 24 hours after the acute therapy to assess infarction size and hemorrhagic status. SICH was defined according to the European Cooperative Acute Stroke Study (ECASS) as a documented hemorrhage associated with a decline $>4$ points in the NIHSS score. Embolic complication was defined as an angiographic occlusion in a previously unaffected vascular territory observed on the angiography after clot removal and associated with new ischemic changes on 24-hour CT or MR imaging.

\section{Intravenous Thrombolysis}

Eligibility for IVT and EVT was evaluated at each center according to the American Heart Association/American Stroke Association guidelines. ${ }^{12}$

\section{MT Approach and ECL Management}

Each center independently decided on the type of approach (i.e., antegrade or retrograde), the ECL treatment (i.e., angioplasty alone, thrombo-aspiration only, or emergency stenting), and the MT technique (i.e., aspiration and/or stent retriever) according to individual operator experience. In the antegrade approach, the highly stenotic ECL that could not be initially broken through was primarily treated and then intracranial MT was performed. In the retrograde approach, after intracranial reperfusion was achieved, ECL was eventually managed. In both antegrade and retrograde approaches, the choice of emergency carotid stent placement was decided on a case-by-case basis, but if a reocclusion occurred in the minutes following intracranial treatment, this method was used at all of the treatment centers in case of a flow-limiting lesion, defined as intracranial ipsilateral or intracranial venous drainage delayed $<2$ seconds compared with the contralateral carotid injection..$^{18}$ The choice of stent type was in accordance with each operator's experience.

In case of emergency stenting placement, the choice of antiplatelet medication was at the discretion of the operators and included single antiplatelet therapy defined as a regular dosage of aspirin (Bayer) (i.e., up to $300 \mathrm{mg}$ ) or a dose of $500 \mathrm{mg}$ of intravenous lysine acetylsalicylate administered before carotid stent. Continuation of antiplatelet therapy was discussed, depending on hemorrhagic transformation detected by 24-hour CT imaging. If no sICH occurred, dual antiplatelet therapy was administered for 3 months. In the case of major hemorrhagic transformation (parenchymal hematoma-1, parenchymal hematoma-2), antiplatelet therapy was stopped at the acute phase. Depending on the follow-up CT or MRI during the postoperative period, the antiplatelet therapy restart was discussed by the medical team.

\section{Statistical Analysis}

Continuous variables were reported as mean \pm SD or median with range and categorical variables as number and percentage. Contingency analyses for categorical variables were performed using the exact chi-square test. Bivariate analysis was used in testing hypotheses of association. Student t-test group comparisons and univariate logistic regression analyses were performed to test differences among the study groups and identify factors influencing clinical outcomes. A 2 -sided $p$ value $<0.05$ was considered to indicate statistical significance. Analyses were performed using IBM SPSS version 27 (IBM Corp.).

\section{Results \\ Study Population}

Between January 2018 and December 2019, 1080 patients admitted with AIS underwent EVT, of whom 214 (19.8\%) presented with a TO. Overall characteristics of the study population are presented in Supplemental Table 1. The median baseline ASPECTS was 8, and the baseline NIHSS score was 17. Bridging IVT/MT was used to treat 101 patients $(52.6 \%)$.

The median onset-to-groin time was $230 \pm 157.7$ minutes, the median onset-to-recanalization time was $300 \pm$ 161.6 minutes, and the median procedural time was $63 \pm$ 55.9 minutes. An antegrade approach was used in 109 of 211 patients $(51.7 \%)$ with complete data available, while 102 patients $(48.3 \%)$ were treated with a retrograde approach. In 156 of 201 patients (77.6\%), there was a CArelated TO, while 45 patients $(22.4 \%)$ presented with a CD-related TO. In 37 patients $(37 / 214 ; 17.3 \%)$, the ECL was treated with angioplasty alone, in 82 patients $(82 / 214$; $38.3 \%$ ) with thrombo-aspiration only, and in 95 patients $(95 / 214 ; 44.4 \%)$ with emergency carotid stenting.

\section{Endovascular Approach}

The antegrade approach was more commonly used in CA-related TOs, and the retrograde approach was mostly used in CD-related TOs. Internal carotid angioplasty was more frequently used in the antegrade than the retrograde approach, and emergency stenting was more frequently used with the retrograde approach than the antegrade approach. Overall characteristics of the subgroups with different technical approaches are presented in Supplemental Table 2.

\section{Clinical and Angiographic Outcomes}

At 3 months poststroke, 86 of 192 patients (44.8\%) were functionally independent, 106 of 192 (55.2\%) had achieved a favorable outcome, and the mortality rate was $21.9 \%$. An mTICI score of $2 \mathrm{~b}-3$ was achieved in 162 of 208 patients (77.9\%) and a TICI score of 3 in 108 of 208 patients (51.9\%). The rate of unsuccessful recanalization was $22.1 \%$. The overall rate of postprocedural intracranial hemorrhage (ICH) was 35\%, and 23 of 214 patients (10.7\%) had sICH, with no differences among CA- and CD-related TOs.

\section{Factors Influencing Clinical Outcome}

Bivariate correlations and odds ratios (ORs) of factors influencing functional independence at 3 months are presented in Table 1. 
TABLE 1. Subgroup comparison of functional dependence and functional independence

\begin{tabular}{|c|c|c|c|}
\hline \multirow[b]{2}{*}{ Patient Characteristics \& Outcomes } & \multicolumn{2}{|c|}{ Functional Status } & \multirow[b]{2}{*}{$\mathrm{p}$ Value } \\
\hline & Dependent & Independent* & \\
\hline Age, yrs & $+0.332(71 \pm 11.9)$ & $-0.332(62.5 \pm 13.3)$ & $<0.001$ \\
\hline Lt hemisphere stroke & +0.046 & -0.046 & 0.52 \\
\hline Baseline NIHSS score & $+0.365(18 \pm 6.3)$ & $-0.365(14.5 \pm 6)$ & $<0.001$ \\
\hline Baseline ASPECTS & $-0.246(8 \pm 1.7)$ & $+0.246(9 \pm 1.5)$ & 0.001 \\
\hline Smoking & +0.024 & -0.024 & 0.74 \\
\hline Diabetes mellitus & +0.137 & -0.137 & 0.43 \\
\hline Dyslipidemia & 0 & 0 & 0.99 \\
\hline Atrial fibrillation & +0.142 & -0.142 & 0.053 \\
\hline Hypertension & +0.150 & -0.150 & 0.04 \\
\hline Prior TIA & +0.023 & -0.023 & 0.75 \\
\hline Onset-to-groin time & -0.106 & +0.106 & 0.18 \\
\hline Onset-to-recanalization time & -0.53 & +0.53 & 0.49 \\
\hline Procedural time & +0.042 & -0.042 & 0.59 \\
\hline MT attempts & $+0.204(2.5 \pm 2.6)$ & $-0.204(2 \pm 1.5)$ & 0.01 \\
\hline $\mathrm{mTICl}$ score $\geq 2 \mathrm{~b}$ & $0.73(0.6-0.9)$ & $3.1(1.6-6.1)$ & $<0.001$ \\
\hline $\mathrm{mTICl}$ score 3 & $0.5(0.4-0.7)$ & $2.2(1.5-3.2)$ & $<0.001$ \\
\hline Preprocedural antiplatelet therapy & +0.073 & -0.073 & 0.31 \\
\hline Intraprocedural antiplatelet therapy & -0.071 & -0.071 & 0.33 \\
\hline Preprocedural anticoagulation therapy & +0.117 & -0.117 & 0.11 \\
\hline Intraprocedural anticoagulation therapy & +0.053 & -0.053 & 0.47 \\
\hline Intravenous thrombolysis & +0.021 & -0.021 & 0.79 \\
\hline Presence of anterior communicating artery & -0.121 & +0.121 & 0.13 \\
\hline Contralateral significant ICA stenosis & -0.013 & +0.013 & 0.86 \\
\hline Antegrade/retrograde approach & $1.4(1.1-1.8)$ & $0.6(0.4-0.9)$ & 0.008 \\
\hline Dissection/atherosclerosis-related ICA occlusion & $0.7(0.5-0.9)$ & $1.5(1.1-2.0)$ & 0.03 \\
\hline ICA angioplasty only & +0.006 & -0.006 & 0.93 \\
\hline ICA aspiration only & +0.082 & -0.082 & 0.25 \\
\hline ICA acute stenting & -0.117 & +0.117 & 0.10 \\
\hline Symptomatic intracerebral hemorrhage & $+0.239(18 / 106)$ & $-0.239(2 / 86)$ & $<0.001$ \\
\hline Intracerebral hemorrhage & $+0.251(49 / 106)$ & $-0.251(19 / 86)$ & $<0.001$ \\
\hline
\end{tabular}

Patients who were functionally independent were younger (median age 61.8 vs 70.6 years), with lower baseline NIHSS scores (median NIHSS score 14.5 vs 18), higher ASPECTS (median ASPECTS 9 vs 8), and lower prevalence of hypertension than functionally dependent patients. Successful intracranial recanalization (mTICI scores $2 b-3$ ) and a TICI score of 3 were associated with more chances of being independent at 90 days, with ORs of 3.1 and 2.2 , respectively.

Overall, patients treated with an antegrade approach had lower likelihood of being functionally independent at 90 days compared with patients treated with a retrograde approach (OR 0.6, 95\% CI 0.4-0.9). Characteristics of the subgroups of patients according to the technical approach are presented in Supplemental Table 2.
Patients with CD-related TO more often achieved 90day clinical independence (OR 1.4, 95\% CI 1.1-2.0) than patients with CA-related TO. There was no difference in sICH rate among patients with CD-related or CA-related ECL occlusion. Characteristics of the subgroups of patients with CD- or CA-related ECL occlusion are presented in Supplemental Table 3. In patients with CD-related ECL occlusion, factors correlated with functional independence were lower age, lower baseline NIHSS score, shorter procedural time, lower number of MT attempts, lower NIHSS score at 24-48 hours, no ICH, and successful intracranial recanalization.

In patients with CA-related ECL occlusion, factors correlated with functional independence were lower age, lower baseline NIHSS score, higher ASPECTS, retro- 
grade approach, no ICH, lower NIHSS score at 24-48 hours, emergency ECL stenting $(\mathrm{p}=0.03$; OR 1.4, 95\% CI 1.1-2.1), and successful intracranial recanalization. On the contrary, patients with CA-related ECL occlusion treated only with aspiration were more often functionally dependent at the 90-day follow-up $(p=0.01$; OR 2.03, 95\% CI 1.1-3.6) (see Supplemental Table 4).

All of the other factors investigated were not associated with good clinical outcomes.

\section{Discussion}

\section{CA-Related Versus CD-Related TO Outcomes}

To date, the different etiologies of ECL in AIS patients with TOs undergoing EVT have been taken into account and compared only in the TITAN and MR CLEAN registries. ${ }^{8,9}$ However, discrepancies in 3-month outcomes were found between CA- and CD-related TOs in these two registries, and further evaluations are needed. In our observational study, successful EVT was more strongly associated with a higher rate of 3-month functional independence in patients with CD-related than those with CA-related TOs. This finding differed from the previously reported data of the TITAN registry, which indicated that patients with internal CA- or CD-related TOs had similar outcomes after successful EVT. ${ }^{8}$ On the other hand, our results are consistent with those obtained from both the MR CLEAN registry ${ }^{9}$ and a systematic review, ${ }^{19}$ which confirmed that a larger proportion of patients with CD-related TOs achieve functional independence following EVT than patients with CA-related TOs. Factors associated with better outcomes in CD-related TOs in our study included younger age, lower burden of cardiovascular risk factors (i.e., diabetes and dyslipidemia), and a lower rate of significant contralateral stenosis of the internal carotid artery (see Supplemental Table 2). Our findings seem to confirm that different etiologies underlying ECL could lead to different prognoses in patients with TOs. This issue indicates that more attention to selection criteria is needed for future trials dealing with TOs.

\section{EVT Strategy: Antegrade Versus Retrograde Approach}

At present, data regarding antegrade versus retrograde approaches during EVT of TOs in the current literature are limited. ${ }^{20}$ In a meta-analysis of CA- and CD-related TOs, no differences in reperfusion rates and clinical outcomes were observed between the antegrade and retrograde EVTs. ${ }^{2}$ In line with these findings, no difference in terms of procedural times, number of MT passages, time to recanalization, and final mTICI score were observed between the two approaches in our study. These results confirm those of the TITAN cohort and other large series. ${ }^{21-23}$ However, we found that patients treated with an antegrade approach had less chance of being independent at 3 months compared with patients with retrograde EVT (see Supplemental Table 3). This result seems to highlight the importance of choosing the best approach based on the ECL etiology underlying TOs, as previously suggested by Marnat et al. ${ }^{24}$ Furthermore, we observed that CA-related TOs were more frequently treated with an antegrade approach and ECL angioplasty, reflecting the frequent tech- nical challenge of passing the extracranial carotid artery obstruction $^{9}$ of an atheromatic lesion compared with performing dissections. Finally, we observed that stenting was mostly used with the retrograde rather than antegrade approach in our series. This result can be justified by the fact that the decision to perform emergency stenting is intrinsically linked to intracranial success. In fact, in case of intracranial reperfusion failure, clinicians would obviously be reluctant to perform emergency stenting considering the drawbacks (e.g., a potentially risky endovascular maneuver with limited expected benefit, probability of extended infarction, and requirement for antiplatelet therapy). Consideration of the deployment of emergency stenting seems reasonable only when the intracranial vessel is reopened.

\section{Emergency Stenting}

Whether the extracranial carotid lesion should be stented or not is controversial. Some authors considered stenting in TOs to be potentially hazardous, ${ }^{25}$ while others tend to perform carotid artery stenting immediately after successful thrombectomy. ${ }^{26}$ In a study recently reported by our group, we found that emergency stenting is safer in defined circumstances, including intraprocedural heparin avoidance, baseline ASPECTS $>8$, and a straightforward MT with a first-pass complete recanalization. ${ }^{27}$ In the present study, we confirmed the safety and efficacy of emergency stenting in TOs irrespective of ECL etiology, without differences in terms of sICH risk between the two groups. However, we found that only patients with CA-related TOs showed a better 3-month outcome after emergency stenting. Thus, as for the previously mentioned choice of EVT approach depending on the ECL that underlies the TOs and also for emergency stenting, its use should be subordinate to ECL etiology and is not necessary for all types of TOs. In accordance with other authors, ${ }^{23,28}$ a more conservative approach may be considered for dissection in case of good collaterals, especially considering the potential of healing of the dissected ECL over time. While the ongoing TITAN randomized trial ${ }^{14}$ and the EASI-TOC ${ }^{15}$ will substantially contribute to the knowledge gained by evaluation of the effect of emergency stenting in TOs, further studies are needed to confirm our results.

\section{Study Limitations}

Our study had several limitations, including the retrospective, noncontrolled design, with individual selection protocols (which may have introduced selection bias) for each participating center. Moreover, the EVT strategy was left to the interventionist's discretion, with heterogeneous ECL treatment modalities employed. Furthermore, the antiplatelet-anticoagulation regimen may have influenced the clinical prognosis in TO. The EVT strategy and antiplatelet therapy decisions were left to the interventionists and may have differed among centers. As reported, this kind of retrospective case series is subject to the biases inherent to this form of uncontrolled, retrospective study. Despite the single and/or dual antiplatelet regimens included in this study, which reflect current standards of 
treatment before carotid artery stenting, further randomized controlled trials are needed to define the best medical therapy to reduce the rate of in-stent restenosis as well as postprocedural intraparenchymal hemorrhage. Although only experienced neuroradiologists assessed data at each institution, we did not confirm our findings using a core laboratory assessment of brain imaging. Finally, despite the effort to establish a comprehensive multicenter registry, there may be a concerning risk of bias due to patients lost to follow-up and missing data in the retrospective data set. Conversely, the strengths of the study included the multicenter design, the use of contemporary technology in a real-world practice, and the fact that this is one of the largest described series of TO patients undergoing MT with a distinction between the two main ECL etiologies underlying TOs.

\section{Conclusions}

Currently, only limited data are available to guide the management of TOs in patients with AIS, and optimal EVT remains to be defined. Our data suggest that the ECL etiology significantly impacts both EVT and outcome in patients presenting with AIS and TOs. A more favorable 3-month clinical outcome should be expected in patients with CD-related TOs compared with CA-related TOs, and this should be taken into account in the decision-making regarding intra- and postprocedural management of patients. Moreover, the retrograde EVT can be desirable in TOs regardless of the ECL etiology. Despite emergency stenting having been confirmed to be safe in both CA- and CD-related TOs, patients with CA-related TOs can gain the most benefit from its use in terms of 3-month clinical outcome. This large multicenter experience provides further refinement of the management of patients with TOs, but controlled studies are warranted to validate these findings and provide additional evidence for clinical practice.

\section{References}

1. Rha JH, Saver JL. The impact of recanalization on ischemic stroke outcome: a meta-analysis. Stroke. 2007;38(3):967-973.

2. Wilson MP, Murad MH, Krings T, et al. Management of tandem occlusions in acute ischemic stroke-intracranial versus extracranial first and extracranial stenting versus angioplasty alone: a systematic review and meta-analysis. J Neurointerv Surg. 2018;10(8):721-728.

3. Engelter ST, Rutgers MP, Hatz F, et al. Intravenous thrombolysis in stroke attributable to cervical artery dissection. Stroke. 2009;40(12):3772-3776.

4. Arauz A, Hoyos L, Espinoza C, et al. Dissection of cervical arteries: long-term follow-up study of 130 consecutive cases. Cerebrovasc Dis. 2006;22(2-3):150-154.

5. Eker OF, Panni P, Dargazanli C, et al. Anterior circulation acute ischemic stroke associated with atherosclerotic lesions of the cervical ICA: a nosologic entity apart. AJNR Am J Neuroradiol. 2017;38(11):2138-2145.

6. Anadani M, Spiotta A, Alawieh A, et al. Effect of extracranial lesion severity on outcome of endovascular thrombectomy in patients with anterior circulation tandem occlusion: analysis of the TITAN registry. J Neurointerv Surg. 2019; 11(10):970-974.

7. Marnane M, Ni Chroinin D, Callaly E, et al. Stroke recurrence within the time window recommended for carotid endarterectomy. Neurology. 2011;77(8):738-743.
8. Gory B, Piotin M, Haussen DC, et al. Thrombectomy in acute stroke with tandem occlusions from dissection versus atherosclerotic cause. Stroke. 2017;48(11):3145-3148.

9. Compagne KCJ, Goldhoorn RB, Uyttenboogaart M, et al. Acute endovascular treatment of patients with ischemic stroke from intracranial large vessel occlusion and extracranial carotid dissection. Front Neurol. 2019;10:102.

10. Berkhemer OA, Borst J, Kappelhof M, et al. Extracranial carotid disease and effect of intra-arterial treatment in patients with proximal anterior circulation stroke in MR CLEAN. Ann Intern Med. 2017;166(12):867-875.

11. Khoury NN, Darsaut TE, Ghostine J, et al. Endovascular thrombectomy and medical therapy versus medical therapy alone in acute stroke: a randomized care trial. J Neuroradiol. 2017;44(3):198-202.

12. Powers WJ, Rabinstein AA, Ackerson T, et al. Guidelines for the early management of patients with acute ischemic stroke: 2019 update to the 2018 Guidelines for the Early Management of Acute Ischemic Stroke: a guideline for healthcare professionals from the American Heart Association/American Stroke Association. Stroke. 2019;50(12):e344-e418.

13. Turc G, Bhogal P, Fischer U, et al. European Stroke Organisation (ESO)-European Society for Minimally Invasive Neurological Therapy (ESMINT) guidelines on mechanical thrombectomy in acute ischaemic strokeendorsed by Stroke Alliance for Europe (SAFE). Eur Stroke J. 2019;4(1):6-12.

14. Zhu F, Hossu G, Soudant M, et al. Effect of emergent carotid stenting during endovascular therapy for acute anterior circulation stroke patients with tandem occlusion: a multicenter, randomized, clinical trial (TITAN) protocol. Int J Stroke. 2021;16(3):342-348.

15. Poppe AY, Jacquin G, Stapf C, et al. A randomized pilot study of patients with tandem carotid lesions undergoing thrombectomy. J Neuroradiol. 2020;47(6):416-420.

16. Ferguson GG, Eliasziw M, Barr HW, et al. The North American Symptomatic Carotid Endarterectomy Trial: surgical results in 1415 patients. Stroke. 1999;30(9):1751-1758.

17. Downer J, Nadarajah M, Briggs E, et al. The location of origin of spontaneous extracranial internal carotid artery dissection is adjacent to the skull base. J Med Imaging Radiat Oncol. 2014;58(4):408-414.

18. Abud DG, Spelle L, Piotin M, et al. Venous phase timing during balloon test occlusion as a criterion for permanent internal carotid artery sacrifice. AJNR Am J Neuroradiol. 2005;26(10):2602-2609.

19. Hoving JW, Marquering HA, Majoie CBLM. Endovascular treatment in patients with carotid artery dissection and intracranial occlusion: a systematic review. Neuroradiology. 2017; 59(7):641-647.

20. Haussen DC, Turjman F, Piotin M, et al. Head or neck first? Speed and rates of reperfusion in thrombectomy for tandem large vessel occlusion strokes. Intervent Neurol. 2020;8(2-6): 92-100.

21. Eker OF, Bühlmann M, Dargazanli C, et al. Endovascular treatment of atherosclerotic tandem occlusions in anterior circulation stroke: technical aspects and complications compared to isolated intracranial occlusions. Front Neurol. 2018;9:1046.

22. Wallocha M, Chapot R, Nordmeyer H, et al. Treatment methods and early neurologic improvement after endovascular treatment of tandem occlusions in acute ischemic stroke. Front Neurol. 2019;10:127.

23. Zhu F, Bracard S, Anxionnat R, et al. Impact of emergent cervical carotid stenting in tandem occlusion strokes treated by thrombectomy: a review of the TITAN collaboration. Front Neurol. 2019;10:206.

24. Marnat G, Bühlmann M, Eker OF, et al. Multicentric experience in distal-to-proximal revascularization of tandem occlusion stroke related to internal carotid artery dissection. AJNR Am J Neuroradiol. 2018;39(6):1093-1099. 
25. Sivan-Hoffmann R, Gory B, Armoiry X, et al. Stent-retriever thrombectomy for acute anterior ischemic stroke with tandem occlusion: a systematic review and meta-analysis. Eur Radiol. 2017;27(1):247-254.

26. Poppe AY, Jacquin G, Roy D, et al. Tandem carotid lesions in acute ischemic stroke: mechanisms, therapeutic challenges, and future directions. AJNR Am J Neuroradiol. 2020;41(7): $1142-1148$.

27. Da Ros V, Scaggiante J, Sallustio F, et al. Carotid stenting and mechanical thrombectomy in patients with acute ischemic stroke and tandem occlusions: antithrombotic treatment and functional outcome. AJNR Am J Neuroradiol. 2020;41(11):2088-2093.

28. Marnat G, Lapergue B, Sibon I, et al. Safety and outcome of carotid dissection stenting during the treatment of tandem occlusions: a pooled analysis of TITAN and ETIS. Stroke. 2020;51(12):3713-3718.

\section{Disclosures}

The authors report no conflict of interest concerning the materials or methods used in this study or the findings specified in this paper.

\section{Author Contributions}

Conception and design: Da Ros. Acquisition of data: Scaggiante, Sallustio, Lattanzi, Chaurasia, Bandettini di Poggio, Toscano,

Rolla Bigliani, Ruggiero, Haznedari, Sanfilippo, Finocchi.

Analysis and interpretation of data: Pitocchi. Drafting the article: Da Ros. Critically revising the article: Umana, Floris. Reviewed submitted version of manuscript: Umana, Haznedari. Approved the final version of the manuscript on behalf of all authors: Umana. Statistical analysis: Sgreccia. Administrative/technical/ material support: Sanfilippo. Study supervision: Diomedi, Floris.

\section{Supplemental Information}

\section{Online-Only Content}

Supplemental material is available online.

Supplemental Tables 1-4. https://thejns.org/doi/suppl/10.3171/ 2021.4.FOCUS21111.

\section{Correspondence}

Giuseppe Emmanuele Umana: Cannizzaro Hospital, Trauma Center, Gamma Knife Center, Catania, Italy. umana.nch@ gmail.com. 